Draft Version November 25, 2020

Typeset using LATEX twocolumn style in AASTeX61

\title{
HAZMAT. IV. FLARES AND SUPERFLARES ON YOUNG M STARS IN THE FAR ULTRAVIOLET*
}

\author{
R. O. Parke Loyd,${ }^{1}$ Evgenya L. Shkolnik, ${ }^{1}$ Adam C. Schneider,${ }^{1}$ Travis S. Barman,${ }^{2}$ Victoria S. Meadows, ${ }^{3,4}$ \\ Isabella Pagano, ${ }^{5}$ And Sarah Peacock ${ }^{2}$
}

\footnotetext{
${ }^{1}$ School of Earth and Space Exploration, Arizona State University, Tempe, AZ 85287

${ }^{2}$ Lunar and Planetary Laboratory, University of Arizona, Tucson, AZ 85721 USA

${ }^{3}$ NASA Astrobiology Institute Alternative Earths and Virtual Planetary Laboratory Teams

${ }^{4}$ Department of Astronomy, University of Washington, Seattle, WA, USA

${ }^{5}$ INAF - Osservatorio Astrofisico di Catania, Via S. Sofia 78, 95123, Catania, Italy
}

(Accepted 2018 September 11)

Submitted to ApJ

V

\section{ABSTRACT}

M stars are powerful emitters of far-ultraviolet light. Over long timescales, a significant, possibly dominant, fraction of this emission is produced by stellar flares. Characterizing this emission is critical to understanding the atmospheres of the stars producing it and the atmospheric evolution of the orbiting planets subjected to it. Ultraviolet emission is known to be elevated for several hundred million years after $M$ stars form. Whether or not the same is true of ultraviolet flare activity is a key concern for the evolution of exoplanet atmospheres. Hubble Space Telescope (HST) observations by the HAZMAT program (HAbitable Zones and M dwarf Activity across Time) detected 18 flares on young (40 Myr) early M stars in the Tucana-Horologium association over $10 \mathrm{~h}$ of observations, ten having energy $>10^{30} \mathrm{erg}$. These imply that flares on young M stars are $100-1000 \times$ more energetic than those occurring at the same rate on "inactive," field age $\mathrm{M}$ dwarfs. However, when energies are normalized by quiescent emission, there is no statistical difference between the young and field age samples. The most energetic flare observed, dubbed the "Hazflare," emitted an energy of $10^{32.1} \mathrm{erg}$ in the FUV, 30× more energetic than any stellar flare previously observed in the FUV with HST's COS or STIS spectrographs. It was accompanied by $15,500 \pm 400 \mathrm{~K}$ blackbody emission bright enough to designate it as a superflare $\left(E>10^{33} \mathrm{erg}\right)$, with an estimated bolometric energy of $10^{33.6_{-0.2}^{+0.1}}$ erg. This blackbody emitted $18_{-1}^{+2} \%$ of its flux in the FUV $(912-1700 \AA)$ where molecules are generally most sensitive to photolysis. Such hot superflares in young, early M stars could play an important role in the evolution of nascent planetary atmospheres.

Corresponding author: R. O. Parke Loyd

parke@asu.edu

* Based on observations made with the NASA/ESA Hubble Space Telescope, obtained from the data archive at the Space Telescope Science Institute. STScI is operated by the Association of Universities for Research in Astronomy, Inc. under NASA contract NAS 5-26555. 


\section{INTRODUCTION}

$\mathrm{M}$ stars are extremely relevant to the quest for an understanding of the diversity, evolution, and biological potential of exoplanets. They dominate planetary systems by number and their small masses and radii make their planets comparatively easy to detect and characterize (see Shields et al. 2016 for a recent review). These stars are known for their vigorous flaring, with flares contributing a significant, potentially dominant portion of the far-ultraviolet (FUV) light they emit (Loyd et al. 2018; hereafter L18). This emission has important consequences for planetary atmospheres. FUV emission photolyzes molecules, perturbing the thermochemical equilibrium these atmospheres would otherwise achieve (e.g., Hu et al. 2012; Miguel \& Kaltenegger 2014). Extreme ultraviolet (EUV) emission, formed alongside the FUV in the stellar upper atmosphere, powers thermal atmospheric escape (e.g., Lammer et al. 2003). Such escape could significantly modify or even entirely remove the primordial atmosphere of a closely orbiting rocky planet.

For M stars of both early (Shkolnik \& Barman 2014) and mid-late (Schneider \& Shkolnik 2018) subtypes, average UV and X-ray emission stays elevated for at least 100 Myr after formation, followed by a decline. Because UV and X-ray emission is magnetically controlled (even if not magnetically sourced; e.g., Hall 2008), a reasonable expectation is that times of higher average emission will also correspond to greater rates of flares. For whitelight flares, this expectation is supported by Davenport's (2016) recent flare analysis of lightcurves in the Kepler archive. This analysis revealed a $t^{-1}$ power-law decay in flare activity with Rossby number, implying that the stellar spin down that occurs with time drives declining flare rates.

This important result from Kepler highlights the usefulness of the recent rise of staring observations, generally intended for detecting signatures of exoplanets, in constraining stellar flares. Kepler lightcurves were also analyzed by Hawley et al. (2014) for M dwarfs classified as active and inactive via $\mathrm{H} \alpha$ emission, concluding that active $\mathrm{M}$ dwarfs flare more frequently than their inactive (but also earlier subtype) counterparts. However, the distinction between groups in flare rates was less distinct than in $\mathrm{H} \alpha$ equivalent widths.

Davenport et al. (2016) used MOST data to determine the flare frequency distribution (FFD, the relationship between the energy and occurrence rate of flares) for the host of the nearest habitable-zone planet, Proxima Centauri, showing that superflares $\left(E_{b o l}>10^{33} \mathrm{erg}\right)$ could occur as often as $8 \mathrm{yr}^{-1}$. Additional data from the Evryscope time-domain survey recently detected one such flare on Proxima Centauri in $1344 \mathrm{~h}(\approx 2$ months $)$ of data (Howard et al. 2018). Assuming an associated particle event, Howard et al. (2018) concluded the planet, if bearing an Earth-like atmosphere, could have experienced substantial destruction of the ozone column. What these optical and infrared photometry surveys detect is primarily the continuum (and "conundruum;" Kowalski et al. 2013) emission from the flares. Continuum emission can vary considerably in temperature, as shown by Kowalski et al. (2013) in an analysis of $U$-band spectra yielding blackbody fits spanning temperatures of 9,000-14,000 K.

Observations of flares at UV wavelengths directly affecting planetary atmospheres are more costly and accordingly rarer. Until recently, they generally focused on specific objects with established track records of large flares, such as AD Leo (M4; e.g. Hawley et al. 2003). However, Welsh et al. (2007) used GALEX UV data to identify 52 flares among 49 stars and concluded that M0-M5 stars exhibit more energetic flares than M6-M8 types.

More recently, L18 identified flares in FUV Hubble Space Telescope (HST) data collected for the MUSCLES Treasury Survey (France et al. 2016a), using these data to constrain FUV flare rates on M dwarfs and comparing inactive and active stellar samples. They found that FFDs between the two samples were nearly identical when flares were characterized by their equivalent duration rather than absolute energy. Equivalent duration is a metric that physically represents the time the star would have to spend in quiescence to emit as much energy within the bandpass of interest as the flare alone. In essence, it normalizes flare energies by the star's quiescent luminosity in the same band. Hence, the consistency in M-dwarf FUV equivalent duration FFDs likely reflects a close correlation between the processes powering quiescent emission and flare emission. This consistency is convenient for predicting flare rates where only a time-averaged measurement of a star's FUV emission is available. However, L18 had no observations of young M stars to test if this consistency also spans M star ages.

Presently, the collection of a new treasury-scale dataset of FUV HST observations is nearing completion as part of the HAbitable Zones and M dwarf Activity across Time (HAZMAT) program (PI Shkolnik). Earlier phases of the HAZMAT program used archival GALEX data to measure the evolution of X-ray, FUV, and NUV flux across time in early (Shkolnik \& Barman 2014) and mid-late (Schneider \& Shkolnik 2018) M stars. These data were also used to measure FUV and NUV variability of $\mathrm{M}$ stars, revealing greater overall variability in the FUV versus the NUV and an increase in NUV 
variability toward later spectral types (Miles \& Shkolnik 2017). The dedicated HAZMAT HST program aims to gather spectra covering most of the FUV and NUV for groups of stars with three well-constrained ages: the Tucana-Horologium (Tuc-Hor) group at $40 \mathrm{Myr}$ (Kraus et al. 2014; Bell et al. 2015), the Hyades cluster at 650 Myr (Maeder \& Mermilliod 1981; Perryman et al. 1998; Martín et al. 2018), and field stars (several Gyr). Observations of the Tuc-Hor moving group members have been completed, consisting of $12 \mathrm{M}$ stars spanning types M0.0-M2.3.

In this work, we identified flares that occurred on these Tuc-Hor objects during the HAZMAT HST observations. One of these flares was a superflare $\left(E_{\text {bolo }}>10^{33}\right.$ erg, greater than any flare yet observed on the Sun), meriting more detailed scrutiny. In Section 2 we describe the observations and stellar sample. In Section 3 we present the detected flares, power-law fits to the flare distributions, and flare rates tabulated for each star. A discussion follows in Section 4 in which we address the implications of the flare distribution and compare to an equivalent dataset for field $\mathrm{M}$ dwarfs of slightly later spectral type (4.1), discuss the substantial quiescent variations in flux observed between flares (4.2), detail the superflare detected during the observations (4.3), and explore the implications of such a flare for a planetary atmosphere (4.4). Results are summarized in Section 5.

\section{OBSERVATIONS}

The analysis presented here utilized data from $12 \mathrm{M}$ star members of the Tuc-Hor young moving group taken with the Cosmic Origins Spectrograph (COS), a photoncounting FUV and NUV spectrograph aboard HST. The data were collected specifically for the HAZMAT program (program ID 14784, PI Shkolnik). The stars in the sample were identified as members of the Tuc-Hor association using 3D space velocities and youth indicators by Shkolnik et al. (2011, 2012) and Kraus et al. (2014). This association is very young, yet old enough that the circumstellar disks have been dispersed, permitting unobstructed observations. Thus data on this association is valuable to investigations of stellar evolution, such as studies of how stellar activity changes with age (Shkolnik \& Barman 2014). The sample includes spectral types ranging from M0.0-M2.3. This range mimics that of Shkolnik \& Barman (2014) for ease of comparison and ensures that stellar age and not spectral type is the primary independent variable sampled by this survey.

The HAZMAT sample selection process ensured that all stars had confirmed ages and no known visual and spectroscopic binaries. In addition to 3D velocities con- sistent with Tuc-Hor membership, the age of the stars is supported by optical spectra that exhibit $\mathrm{H} \alpha$ in emission without lithium in absorption (lithium is rapidly burned after about $10 \mathrm{Myr}$ for late $\mathrm{K}$ and early M stars; Kraus et al. 2014). Specifically, the lithium depletion boundary in Tuc-Hor objects implies an age of 35-45 Myr for the group, consistent with isochrone fits by Bell et al. (2015) yielding a prediction of $45 \pm 4 \mathrm{Myr}$. We adopt $40 \mathrm{Myr}$ as the age of the group. Table 1 gives selected properties for each of the objects.

For comparison with earlier work, we restrict this analysis to the FUV data taken with COS's G130M grating. The wavelength coverage of this configuration is $\sim 1170-1430 \AA$ and includes strong emission lines of C II, C III, Si III, Si IV, and N V formed in the stellar transition region and a smattering of weaker lines (including some coronal iron lines). Ly $\alpha$ and O I are in the bandpass also, but are typically lost to contamination by telluric emission (geocoronal airglow). Because COS uses a photon-counting detector (the raw data being simply a list of detector position and time for each photon), the flux can be measured in arbitrary bins of wavelength and time to create lightcurves, integrated spectra, and subsampled spectra. While there are instrumental limits to the wavelength and time resolution, in practice signal-to-noise ratio $(\mathrm{S} / \mathrm{N})$ requirements ultimately set much coarser resolution limits.

Most objects in this program were exposed for roughly $1 \mathrm{ks}$, with two exposed for $10 \mathrm{ks}$. To mitigate the effect of fixed-pattern noise on integrated spectra, the observations are dithered, with gaps of about $100 \mathrm{~s}$ between each exposure. For longer total exposures, these gaps instead last the $\sim 45$ minute duration over which a target is obscured by the Earth during HST's orbit. These gaps can obscure portions of flares and introduce ambiguity into whether a single event or multiple events occurred and the total energy of the event(s). The uncertainty this introduces in deriving characteristics of the overall flare population is further addressed in Section 3.

\section{ANALYSIS}

We identified flares in the data using the FLAIIL (FLAre Identification in Interrupted Lightcurves ${ }^{1}$ ) algorithm described by L18, including the same FUV 130 bandpass. Briefly, the method estimates quiescent fluxes using a Gaussian Process model with a covariance kernel of the form $\sigma_{x}^{2} e^{-\Delta t / \tau}$, where $\Delta t$ is the difference in time between data points and $\sigma_{x}^{2}$ and $\tau$ are parameters specifying the variance and decorrelation timescale of the data. The $x$ in $\sigma_{x}^{2}$ denotes that the variations be-

1 https://www.github.com/parkus/flaiil 


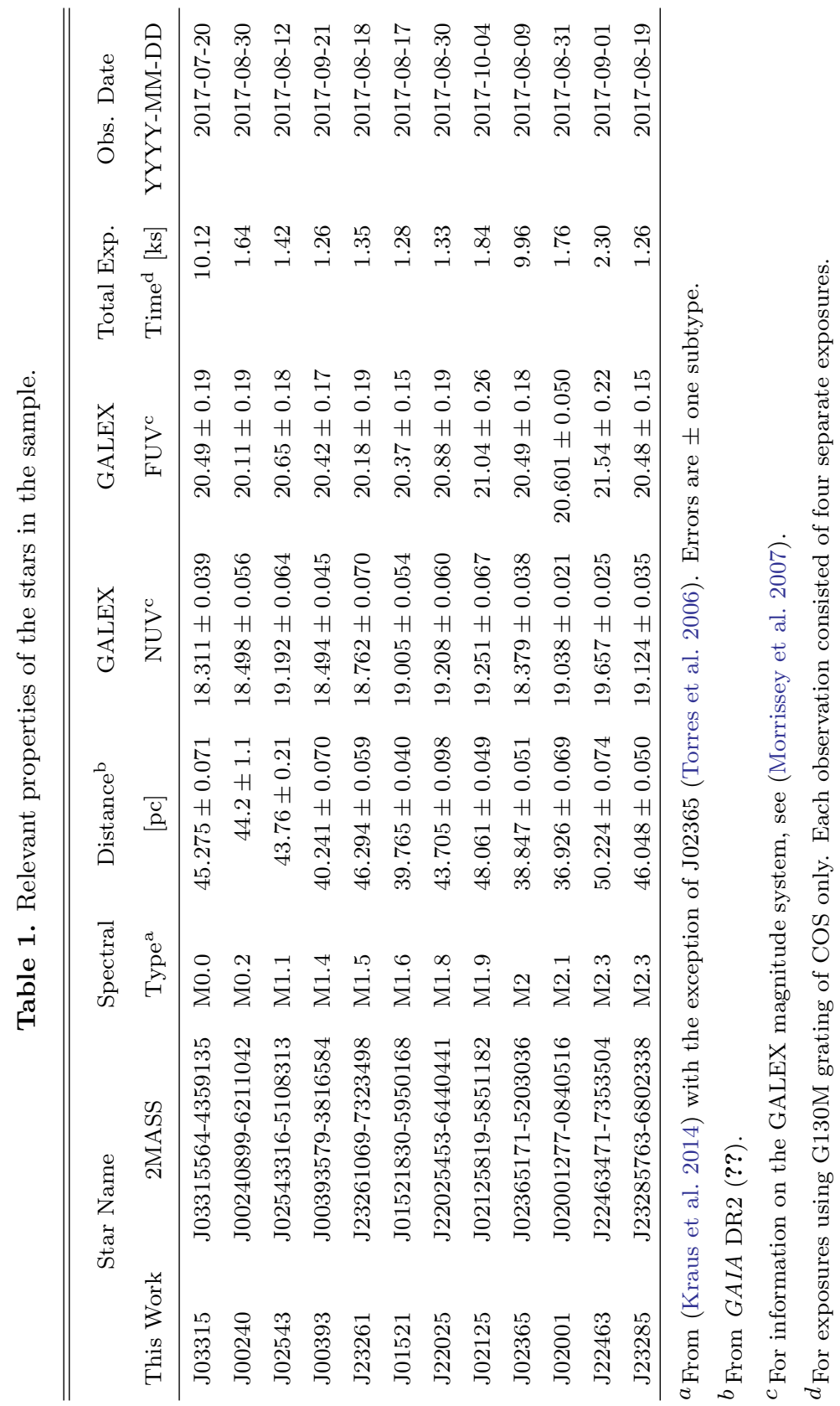


ing fit are those in excess of what would be expected from measurement uncertainty. A penalty is applied for power at $0.1 \mathrm{~Hz}$ to mitigate overfitting of noise and flares and a flat line is used when the likelihood ratio of such a model to the Gaussian process is $<2$.

Following quiescence fitting, the lightcurve is divided into "runs" of points above and below quiescence, and runs with an integrated area $5 \sigma$ above quiescence are flagged as flares and those with $3 \sigma$ above or below quiescence are flagged as suspect. The process is iterated, with each iteration conducting a maximum likelihood fit of the Gaussian Process model to the nonflare and nonsuspect points. Iteration is terminated when the same points are successively flagged as flare or suspect.

A bonus of the identification procedure is that the Gaussian Process fit provides a measure of the amplitude and timescale of the star's quiescent variations. For the young M stars, quiescent variations can be quite significant (Section 4.2). A useful relationship for predicting the expected sample standard deviation, $[S]$, as a function of lightcurve binning, $\Delta t$, based on the Gaussian Process fit is

$$
\left[S_{x}\right]=\left(\frac{2 \sigma_{x}^{2}}{(\Delta t / \tau)^{2}}\left(\Delta t / \tau+e^{-\Delta t / \tau}-1\right)\right)^{1 / 2} .
$$

In addition to quantifying quiescent variations via a Gaussian Process, we compute the 60 s "excess noise" metric as per Loyd \& France (2014) and the relative median absolute deviation (MAD) as per Miles \& Shkolnik (2017) to facilitate comparisons between these works. The results are tabulated in Table 3.

To produce lightcurves, we integrated nearly the same wavelength range as the $\mathrm{FUV}_{130}$ band from L18. This spans most of the COS G130M bandpass, excluding areas contaminated by telluric emission, roughly covering $1170-1270$ and $1330-1430 \AA$. We record the total energy, $E$; equivalent duration, $\delta$; peak flux, $F_{p}$; full width at half max, FWHM; rise time; and decay time for each flare. Rise times, decay times, and the FWHM are made ambiguous by noise fluctuations and complex, multi-peaked structures in some flares. Thus, the rise and decay times are measured as the time between last crossing of the quiescent level and first crossing of half$\max$ at the flare start and vice versa at the flare end. The FWHM is the sum of all time during which the flare's flux was above half-max.

We detected a total of 18 flares in $35.5 \mathrm{ks}$ of exposure of 12 targets with COS G130M. Included in this sample is a flare that emitted $10^{32.1} \mathrm{erg}$ in the FUV, exceeding the most energetic M-star flare previously observed in the FUV with $H S T$ by about a factor of 30 . Because these stars are comparatively distant (38-53 pc) relative to the older, less FUV-luminous M dwarfs previously observed by $H S T$, the smallest detectable flares are correspondingly more energetic. However, their range of equivalent durations, $20-6700 \mathrm{~s}$, is similar to the previously observed flares. The detected flares and their properties are tabulated in Table 2.

For each star, as well as the aggregated sample, we fit power laws to the FFDs. Preferences in the literature vary in exactly how to specify this power law, but in this work we will use the cumulative form

$$
\nu=\mu E^{-\alpha}
$$

where $\nu$ is the frequency of flares with energy (equivalent duration) greater than $E(\delta), \mu$ specifies the rate of flares with unit energy (equivalent duration), and $\alpha$ is the index of the power law.

We fit FFDs in both absolute energy and equivalent duration using the same method used by L18. The method computes a likelihood of the individual event energies (i.e., events are not binned) given a power-law index as well as the Poisson-likelihood of the number of events observed once the rate constant is applied. The MCMC sampler emcee (Foreman-Mackey et al. 2013) is then used to sample the posterior of these parameters. Tables 4 and 5 give the parameters of these fits for each star and the flares aggregated from all stars.

These fits then enable estimates of the rate of flares above various energy and equivalent duration limits. Considering long timescales over which many flares occur, the FFD fits allow predictions of the cumulative energy emitted by flares and the ratio of this energy to that emitted by quiescence. Further, one can estimate the "critical equivalent duration," defined as the limit to which integrating the FFD fit predicts energy emitted by the star's flares will exceed that emitted by the star in quiescence (L18). We used the MCMC samples of the fit parameters to sample the posterior distribution of these derived quantities, thereby accounting for the strong correlation between the fit parameters.

No single star exhibited enough flares $(\gtrsim 5)$ to effectively constrain the index, $\alpha$, of the power law in a fit to the FFD. However, by applying an a priori constraint on this index, the rate constant, $\mu$, of flares can also be constrained. This in turn provides constraints on flare rates and other derived quantities. Hence, we use this technique for the individual stars. For the necessary constraint on the power-law index, we use the posterior probability distribution from the fit to the aggregated flares from all stars as a prior for each individual star. The fits to the aggregated flares are

$$
\nu(E)=10^{1.38_{-0.13}^{+0.12}} \mathrm{~d}^{-1}\left(\frac{E}{10^{30} \mathrm{erg}}\right)^{-0.61_{-0.13}^{+0.15}}
$$


Table 2. Identified flares.

\begin{tabular}{|c|c|c|c|c|c|c|c|c|c|}
\hline \multirow[t]{2}{*}{ Star } & \multirow{2}{*}{$\begin{array}{l}\delta \\
\mathrm{s}\end{array}$} & \multirow{2}{*}{$\begin{array}{c}E \\
10^{30} \mathrm{erg}\end{array}$} & \multirow{2}{*}{$\begin{array}{l}t_{\text {peak }} \\
\text { MJD }\end{array}$} & $F_{\text {peak }}$ & \multirow[t]{2}{*}{$\frac{F_{\text {peak }}}{F_{q}}$} & \multirow{2}{*}{$\begin{array}{c}\text { Rise Time } \\
\text { s }\end{array}$} & \multirow{2}{*}{$\begin{array}{c}\text { FWHM } \\
\mathrm{s} \\
\end{array}$} & \multirow{2}{*}{$\begin{array}{c}\text { Decay Time } \\
\text { s }\end{array}$} & \multirow[t]{2}{*}{ Complex? ${ }^{\mathrm{b}}$} \\
\hline & & & & $10^{-13} \frac{\mathrm{erg}}{\mathrm{cm}^{2} \mathrm{~s} \mathrm{~A}}$ & & & & & \\
\hline \multirow[t]{4}{*}{ J02365 } & $6736 \pm 40$ & $130.64 \pm 0.55$ & 57974.5621 & $66.7 \pm 6.8$ & $63.1 \pm 6.7$ & 150 & 55 & 770 & $\mathrm{Y}$ \\
\hline & $832 \pm 14$ & $16.13 \pm 0.26$ & 57974.3864 & $6.82 \pm 0.80$ & $7.35 \pm 0.89$ & 88 & 37 & 330 & $\mathrm{Y}$ \\
\hline & $359.7 \pm 9.8$ & $6.98 \pm 0.19$ & 57974.4511 & $4.98 \pm 0.59$ & $5.64 \pm 0.69$ & 17 & 65 & 180 & $\mathrm{~N}$ \\
\hline & $48.2 \pm 6.1$ & $0.93 \pm 0.12$ & 57974.4311 & $1.72 \pm 0.22$ & $2.60 \pm 0.34$ & 22 & 53 & 14 & $\ldots$ \\
\hline J01521 & $1961 \pm 45$ & $12.78 \pm 0.19$ & 57982.9865 & $4.43 \pm 0.53$ & $13.9 \pm 2.7$ & 130 & $74^{\mathrm{c}}$ & $\ldots$ & $\ldots$ \\
\hline \multirow[t]{4}{*}{ J03315 } & $405 \pm 14$ & $7.37 \pm 0.26$ & 57954.8492 & $2.68 \pm 0.31$ & $4.65 \pm 0.55$ & 150 & 23 & 75 & $\cdots$ \\
\hline & $101.6 \pm 8.4$ & $2.00 \pm 0.17$ & 57954.9052 & $1.49 \pm 0.18$ & $2.85 \pm 0.34$ & 16 & 53 & 41 & $\ldots$ \\
\hline & $46.0 \pm 7.1$ & $0.77 \pm 0.12$ & 57954.7906 & $1.02 \pm 0.12$ & $2.49 \pm 0.30$ & 3.9 & 93 & 5.1 & $\ldots$ \\
\hline & $40.4 \pm 6.2$ & $0.77 \pm 0.12$ & 57954.8642 & $1.58 \pm 0.17$ & $3.05 \pm 0.34$ & 7.1 & 9.4 & 25 & $\cdots$ \\
\hline J22025 & $300 \pm 14$ & $6.53 \pm 0.21$ & 57995.2908 & $15.1 \pm 1.6$ & $16.8 \pm 2.6$ & 19 & 9.7 & 110 & $\mathrm{~N}$ \\
\hline J02543 & $214 \pm 10$ & $2.26 \pm 0.11$ & 57977.8361 & $1.55 \pm 0.20$ & $4.37 \pm 0.58$ & 26 & . c & $\ldots$ & $\ldots$ \\
\hline J00240 & $96.7 \pm 9.0$ & $1.53 \pm 0.14$ & 57995.8298 & $1.99 \pm 0.25$ & $3.93 \pm 0.67$ & 29 & 8.1 & 7.7 & $\ldots$ \\
\hline $\mathrm{J} 23285$ & $89.9 \pm 9.8$ & $1.23 \pm 0.13$ & 57984.0811 & $1.07 \pm 0.14$ & $2.98 \pm 0.49$ & $\ldots$ & $56^{\mathrm{c}}$ & 12 & $\ldots$ \\
\hline \multirow[t]{4}{*}{ J02001 } & $66.4 \pm 6.3$ & $0.752 \pm 0.071$ & 57996.5731 & $2.14 \pm 0.24$ & $4.08 \pm 0.48$ & 26 & . . c & $\ldots$ & $\ldots$ \\
\hline & $42.0 \pm 6.0$ & $0.480 \pm 0.069$ & 57996.5586 & $1.15 \pm 0.15$ & $2.64 \pm 0.35$ & 9.8 & 52 & 12 & $\cdots$ \\
\hline & $31.7 \pm 4.1$ & $0.356 \pm 0.046$ & 57996.5666 & $1.45 \pm 0.17$ & $3.11 \pm 0.36$ & 51 & $\ldots \quad c$ & $\ldots$ & $\ldots$ \\
\hline & $21.5 \pm 4.5$ & $0.296 \pm 0.062$ & 57996.5068 & $1.46 \pm 0.17$ & $2.73 \pm 0.33$ & 18 & 17 & 9.6 & $\ldots$ \\
\hline J00393 & $40.7 \pm 5.1$ & $0.404 \pm 0.051$ & 58017.9703 & $1.39 \pm 0.16$ & $3.72 \pm 0.43$ & 39 & $15^{\mathrm{c}}$ & $\ldots$ & $\ldots$ \\
\hline
\end{tabular}

${ }^{a}$ Ratio of peak flux to quiescent flux.

${ }^{b}$ Subjective determination of the complexity of the flare shape based on its deviation from an impulse-decay, generally due to multiple peaks. No data indicates the flare was not well-enough resolved or the classification was particularly ambiguous.

${ }^{c}$ Flare cut off by the start or end of an exposure.

Note-Uncertainties are statistical and do not reflect systematic effects due to choices made in the flare identification and measurement algorithm. See text for a discussion of the effect of these choices.

and

$$
\nu(\delta)=10^{0.68_{-0.27}^{+0.23}} \mathrm{~d}^{-1}\left(\frac{\delta}{1000 \mathrm{~s}}\right)^{-0.59_{-0.13}^{+0.15}}
$$

Using the stabilized Kolmogorov-Smirnov test recommended by (Maschberger \& Kroupa 2009), we find that both power laws provide acceptable fits to the data, with $p$-values of 0.6 (energy) and 0.7 (equivalent duration).

It is critical to note that the uncertainties specified throughout this work are statistical. However, systematics, such as the exposure gaps mentioned in Section 2 , and subjective choices made in constructing the flare identification algorithm influence the results. These affect the overall number and measured characteristics (most importantly energy) of the identified flares. We adjusted the parameters of our flare identification and FFD fitting apparatus within reasonable ranges and observed changes to assess the degree of the effect on the FFD power-law index. This includes the readiness with which events closely spaced in time relative to the overall duration are associated, including those broken up by an exposure gap. The appendices of L18 include a more detailed discussion of the various parameters. For this work, we found that the total number of flares varied between 16 and 22 and the index of the equivalent duration FFD fit varied between 0.4 and 0.8 according to our analysis choices. This is important to bear in mind when interpreting FFDs and derived values.

\section{DISCUSSION}

\subsection{Star Flares at 40 Myr are 100-1000× More Energetic than at Field Ages}

The primary question driving this analysis was "how does FUV flare activity on M stars evolve over their lifetimes?" This is answered in Figure 1, in which the FFDs of the 40 Myr stars and the field-age stars are compared, using both equivalent duration and absolute energy. The field-age curve is taken from L18 and relies on observations acquired by the MUSCLES program (France et al. 2016b) of GJ 667C (M1.5), GJ 436 (M2.5), GJ 832 (M2/3), GJ 1214 (M3), GJ 581 (M3), and GJ 876 (M3.5), with ages ranging from 1-9 Gyr (Anglada-Escudé et al. 2013; Torres et al. 2008; Sanz- 
Table 3. Quiescence fit parameters and measurements of quiescent variability.

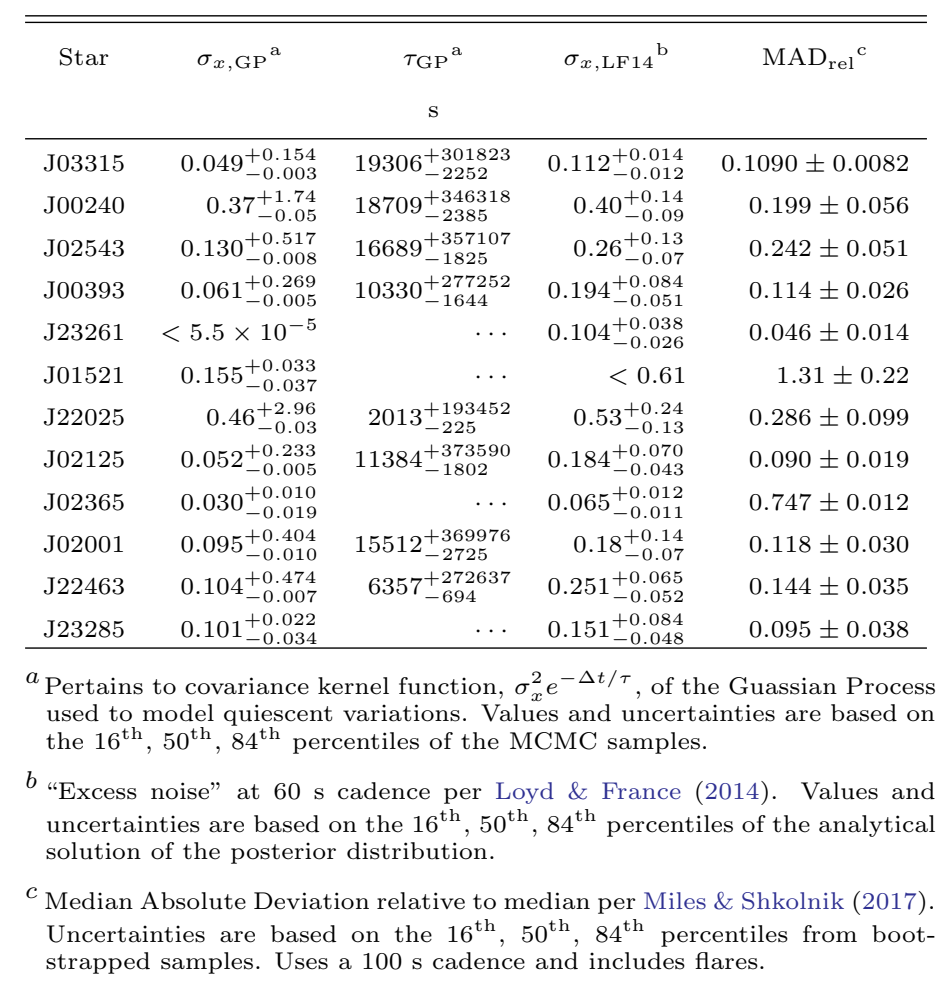

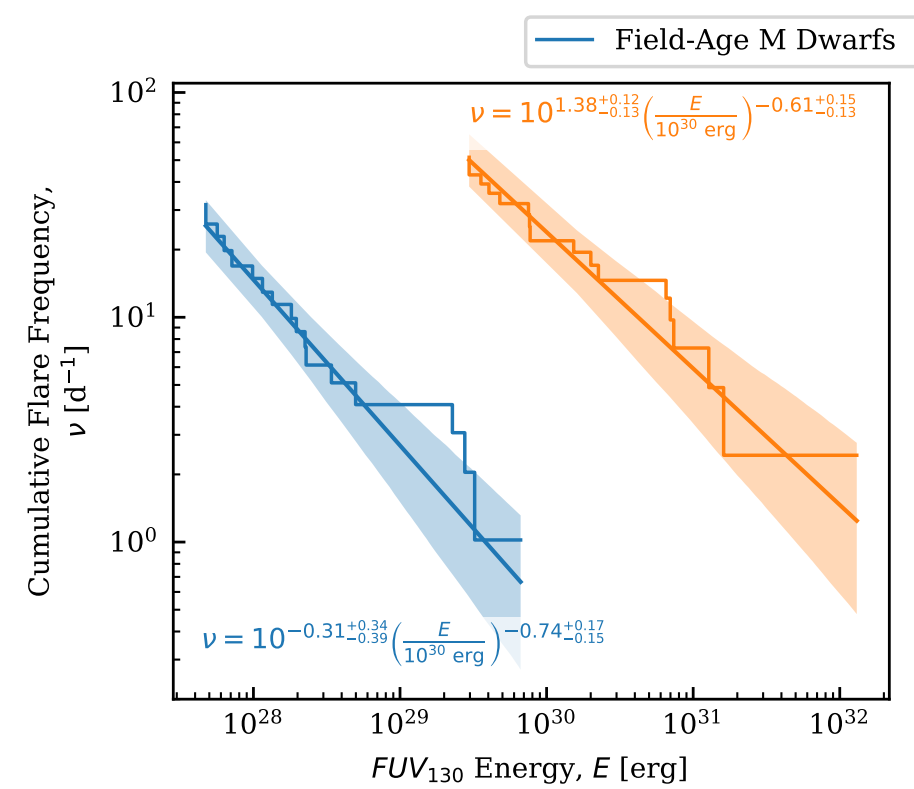

40 Myr M Stars

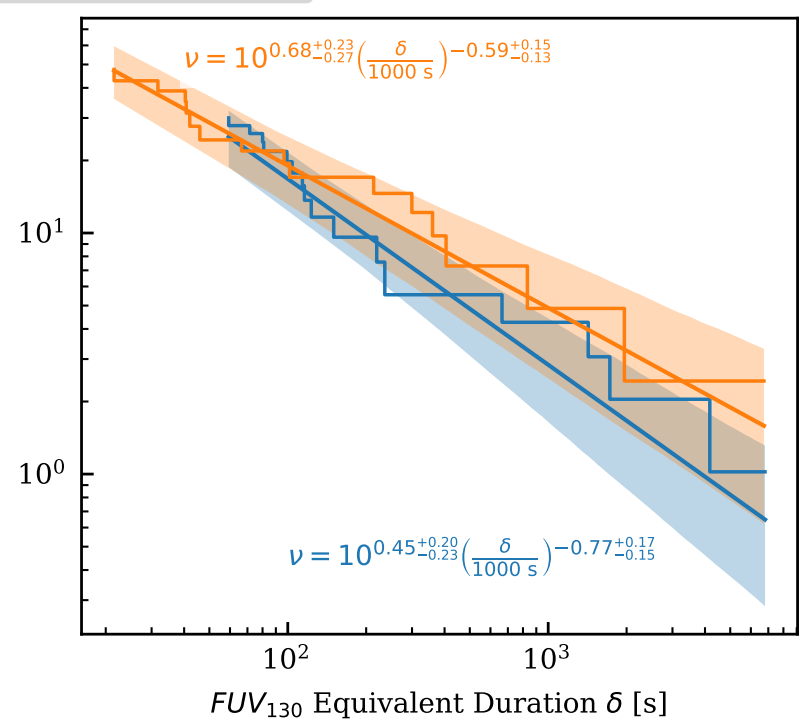

Figure 1. Comparison of the flare frequency distributions (FFDs) of field-age (from L18) and 40 Myr M stars. Stepped lines give the cumulative flare rate (corrected for differing detection limits between observations), straight lines are power-law fits, and shaded regions are $1 \sigma$ errors on those fits. In absolute energy (left), the limiting energy for flares occurring less than 5 times per day is 100-1000× greater on the $40 \mathrm{Myr}$ versus field-age stars. However, in equivalent duration (right) the rates are statistically indistinguishable over the observed range. Note that spectral types differ somewhat, spanning M0.0-M2.3 for the 40 Myr sample and M1.5-M3.5 for the field sample. 


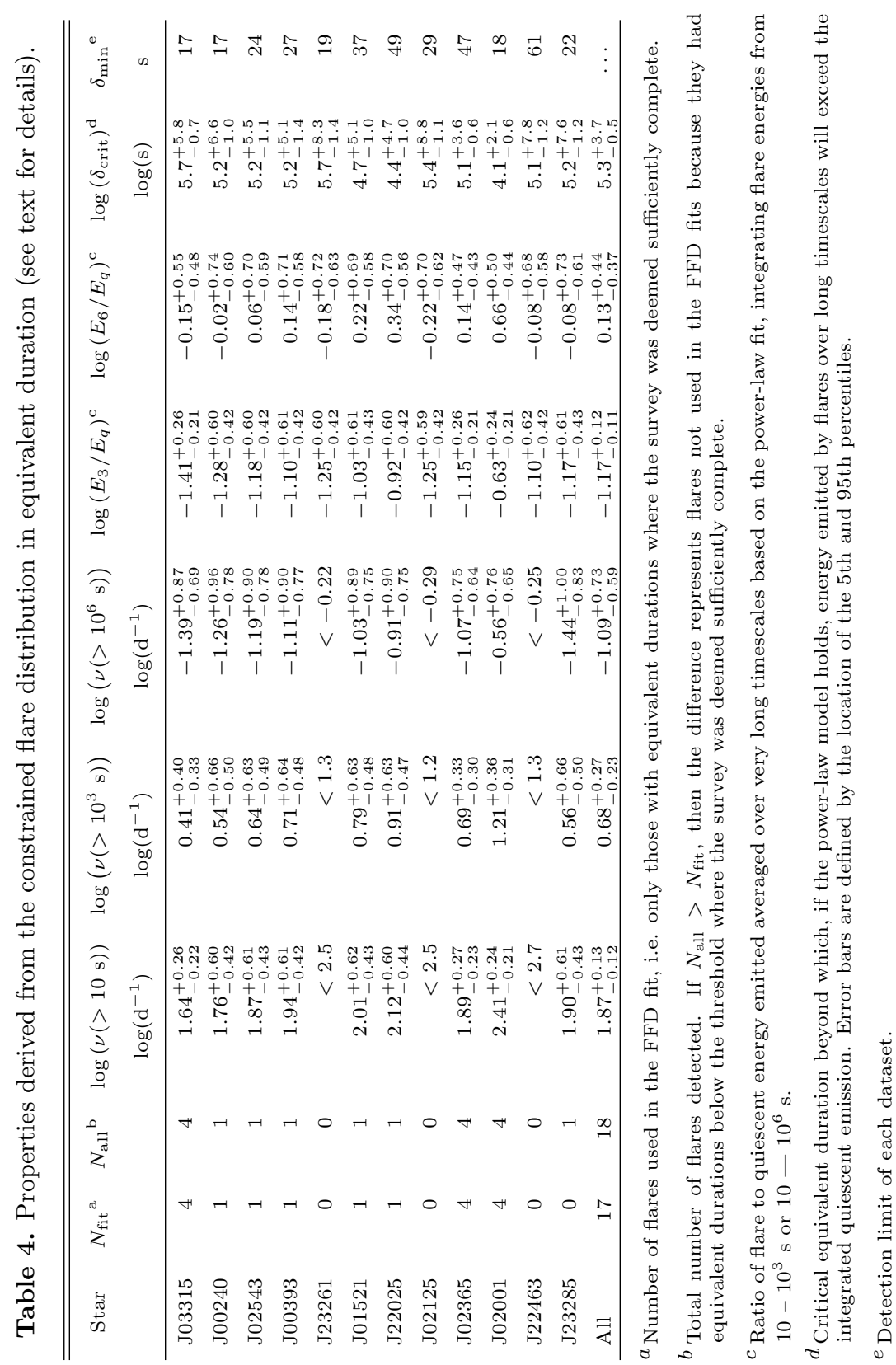




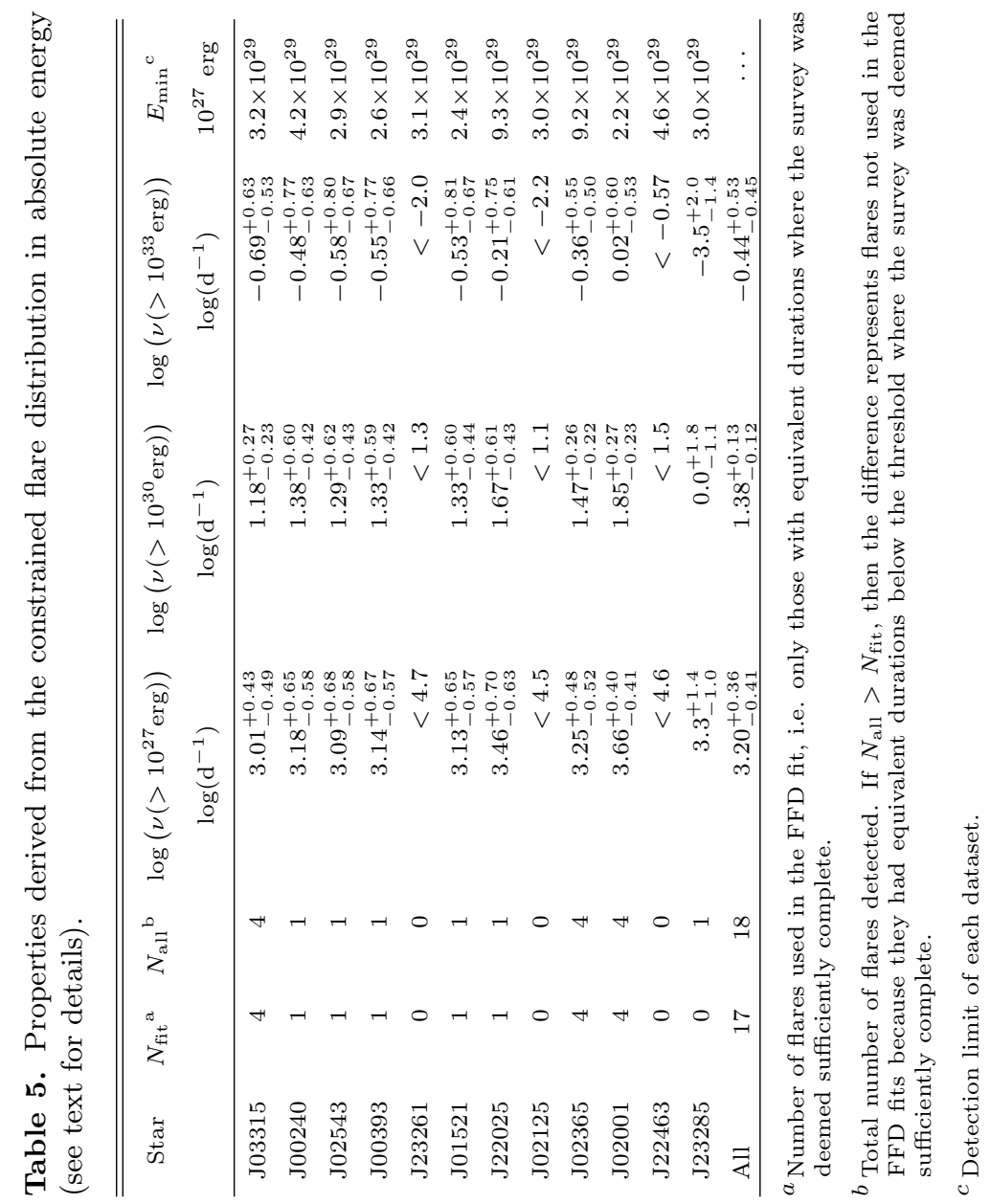


Forcada et al. 2010; Berta et al. 2011; Selsis et al. 2007; Rivera et al. 2010).

The energy above which flares occur less than 5 times per day is $100-1000 \times$ greater for the $40 \mathrm{Myr}$ versus the field-age $\mathrm{M}$ stars. Comparing, instead, the rates of flares with energies $>10^{30} \mathrm{erg}$, those on $40 \mathrm{Myr}$ stars occur $20-100 \times$ more frequently. (Note that quiescent flux over the course of the flare is subtracted when computing energies, so the difference in the absolute energy FFDs is not due simply to integrating different quiescent luminosities.) The FFD of the active star sample from L18 (not plotted to avoid clutter) falls between these two.

The two distributions overlap extensively when flares are characterized by their equivalent duration. This suggests that the conclusion of L18 that all M dwarfs flare similarly in the FUV when flares are characterized by their equivalent duration can be extended to young $M$ stars as well. The difference in the power-law indices implies that this will not hold true beyond the plotted range, but this difference is not statistically significant. Any true difference in the indices and thus rate of higher-energy flares, if it exists, can only be resolved with more variability monitoring of young $M$ stars in the FUV. The similarity of the distributions in equivalent duration means the differences in absolute energy are due almost entirely to the differing quiescent FUV luminosity of the stars.

The stellar samples differ somewhat in their makeup of spectral types. The $40 \mathrm{Myr}$ stars vary from M0.0 to M2.3 while the field-age stars vary from M1.5 to M3.5. The only field-age object with a spectral type confidently within the range of $40 \mathrm{Myr}$ sample is GJ $667 \mathrm{C}$, and the rates of $\delta=1000 \mathrm{~s}$ and $E=10^{30}$ erg flares estimated for this star fall squarely within the rest of the field-age sample. If anything, the differences in spectral types would be expected to lessen the gap in the absolute energy FFDs due to greater flare activity on later-type M dwarfs (Hilton 2011) and create a gap in the equivalent duration FFDs due to differing stellar conditions. Hence, the conclusions regarding the difference in absolute flare energies and similarities in equivalent duration are likely to hold for samples more consistent in spectral type. Upcoming observations for the HAZMAT program will add three field-age M stars with M0.5, M0.5, and M2.0 types, enabling a future flare analysis of a fieldage sample with stellar types in closer alignment with the other age groups sampled by HAZMAT.

The differences in flare activity accord well with the measurements made by Shkolnik \& Barman (2014) and Schneider \& Shkolnik (2018) of changes in FUV and NUV activity over time. Their measurements used observations from GALEX whose FUV bandpass spans
1340-1800 A (containing the strong emission lines Si IV, $\mathrm{C}$ IV, and He II), while the present analysis relies on emission from an 1170-1270 + 1330-1430 A range (containing C III, Si III, N V, C II, Si IV). Shkolnik \& Barman (2014) measure a median GALEX FUV flux for the $40 \mathrm{Myr}$ objects in their sample that is $20 \times$ that of the field objects, similar to the drop in the rate of $>10^{30} \mathrm{erg}$ $\mathrm{FUV}_{130}$ flares measured in this analysis. In other words, it appears quiescent flux levels and the energy output of flares drop in synchrony, further supporting the consistency of M-star equivalent-duration FFDs found by L18. If this similarity between evolution in quiescent flux levels and flare rates holds, then the $650 \mathrm{Myr}$ (Hyades) stars will flare at a slightly reduced (factor of a few) rate relative to the $40 \mathrm{Myr}$ (Tuc-Hor) stars. HST observations of the Hyades cluster for the HAZMAT program will be complete this year.

The uniformity of $\mathrm{M}$ dwarf flares in equivalent duration seems a natural consequence of magnetic activity both heating the stellar transition region during quiescence as well as producing flares. It is evidence against other mechanisms of quiescent heating, such as upwelling shocks (Hall 2008), unless somehow these shocks are linked to flares. This relationship should not to be taken to imply quiescent emission is due simply to unresolved flares. As L18 noted, extrapolating the FFD of $\mathrm{M}$ dwarfs to zero and integrating could only account for a small fraction of quiescent emission (though this is not true for flares in some specific emission lines like Si IV). This means that flare and quiescent FUV emission either arise from distinct mechanisms or the FFDs steepen considerably (and consistently) at unresolved flare energies. Consistency in equivalent duration FFDs does not appear to extend to the Sun, which exhibits FFDs with rates three orders of magnitude below those of $\mathrm{M}$ dwarfs in analogous EUV emission (L18).

Regardless of the source of the consistency in equivalent duration FUV FFDs for M stars, it is a convenient fact. It implies models of stellar flaring, such as those that might be used in assessing the impacts to planetary atmospheres, can utilize an estimate of a star's FUV luminosity as a single parameter to describe the star's FUV flare activity within the range of energies thus far observed.

An important feature of FUV flares on M stars is that they might dominate the energy budget of FUV emission from such stars over timescales long enough to include rare, highly energetic flares not yet observed in the FUV. For field age objects, if the power-law FFD estimated by L18 extends $2-5$ orders of magnitude beyond the most energetic flare identified in that analysis, then flare emission begins to dominate. Considering the 


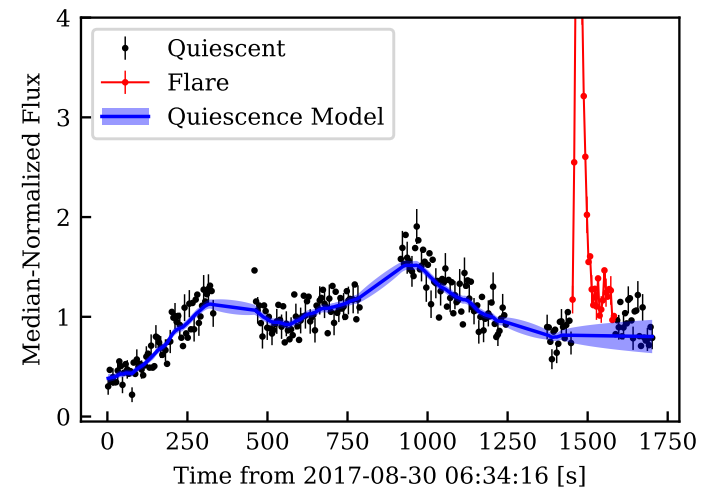

Figure 2. FUV 130 lightcurve of J22025 showing the factor of a few variations during quiescence.

most energetic flare here observed was $30 \times$ more energetic, the likelihood that flares indeed dominate over quiescence is increased. For the distribution of aggregated $40 \mathrm{Myr} \mathrm{M}$ star flares, this limit is $10^{5}-10^{9} \mathrm{~s}$ in equivalent duration $\left(10^{33}-10^{37} \mathrm{erg}\right), 1-5$ orders of magnitude beyond the most energetic event observed.

\subsection{Substantial Quiescent Variability in Young Ms}

FUV emission from the young M stars in this sample exhibits substantial levels of quiescent variability. As part of the analysis, we computed several measures of variability, presented in Table 3. An example of these variations are those of J22025 where the ratio of the maximum to the minimum observed flux during quiescence is 6 . The $\mathrm{FUV}_{130}$ lightcurve of this star is depicted in Figure 2. Although the quiescent variations of J22025 are among the largest in the sample, the values in Table 3 make it clear that significant quiescent variations are normal for the observed stars.

The FUV variability of M stars has also been surveyed by Loyd \& France (2014) and Miles \& Shkolnik (2017). Loyd \& France (2014) conducted an analysis of archival $H S T$ data similar to that conducted here, but using flux from specific emission lines that are major contributors to the flux in the band employed for the present analysis (C II, Si III, Si IV). The most comparable stars in their sample are GJ 832 (M2/3) and AU Mic (M1), a known young flare star that is still contracting toward the main sequence. GJ 832 exhibits "excess noise," $\sigma_{x}$, of $0.1-0.15$ in the major lines, similar to the lower end of the present sample. AU Mic exhibits excess noise $<0.03-0.1$, below all but two of the stars in the present sample.

Miles \& Shkolnik (2017) analyzed archival GALEX data using the MAD relative to the median, MADrel. Only a few points were available to characterize variability, precluding the identification and removal of flares in that work. Therefore, MAD rel measurements for the
40 Myr M stars in Table 3 include flares, though the statistic is mostly insensitive to them. The $40 \mathrm{Myr} \mathrm{M}$ stars exhibit $\mathrm{MAD}_{\text {rel }}$ values within the upper half of the Miles \& Shkolnik (2017) sample, with J01521 and J22025 reaching the upper extreme.

In some cases, the variations exhibit a clear timescale. This is quantified by the decay-timescale for autocorrelations in the Gaussian Process we used to model quiescence. Because a penalty was applied for nonsmoothness (to avoid overfitting noise and potentially "fitting out" flares), decay timescales are systematically longer than otherwise. Timescales range from tens of minutes to hours, with J22025 showing the clearest timescale by eye to its variations. Lifting the nonsmoothness penalty for that object results in a time-constant of $1800_{-1000}^{+4000} \mathrm{~s}$ for the Gaussian Process fit to quiescence. This is too rapid to be attributed to stellar rotation, but matches the expected timescale of convective granulation (Kjeldsen \& Bedding 1995). Therefore, we posit that convective motions are modulating the magnetic heating in one to a few localized active regions in this star and perhaps others exhibiting large-amplitude variations with a clear timescale. The amplitude of the variations suggests a limit to the number of active regions that could be contributing significantly to the quiescent flux. A greater number of independently evolving regions would result in those fluctuations averaging out (unless the fluctuations are of correspondingly larger amplitude). A simple test of this hypothesis is to compare simultaneous optical and FUV observations. If convection is driving quiescent FUV variability, the timescale of optical and FUV fluctuations for a given star should match.

It might seem likely that stars with larger-amplitude quiescent variations would have more tumultuous magnetic heating leading to more flares. Yet the stars exhibiting flares in this sample do not exhibit anomalously high quiescent variability. Alternatively, suppressed quiescent variations might indicate that magnetic energy that would have powered them is instead building toward a catastrophic release in the form of a flare, i.e. that smaller-amplitude quiescent variations would be accompanied by more frequent flares. Yet this too is not apparent in the data. Indeed, the stars exhibiting flares span nearly the full range of quiescent variability. Future datasets providing longer baselines, particularly from staring NUV and FUV observations by the upcoming SPARCS (Shkolnik 2016) and CUTE (Fleming et al. 2018) cubesats, could determine whether quiescent variations and flares are truly uncorrelated.

\subsection{Hubble FUV Spectrophotometry of an M Star Superflare}




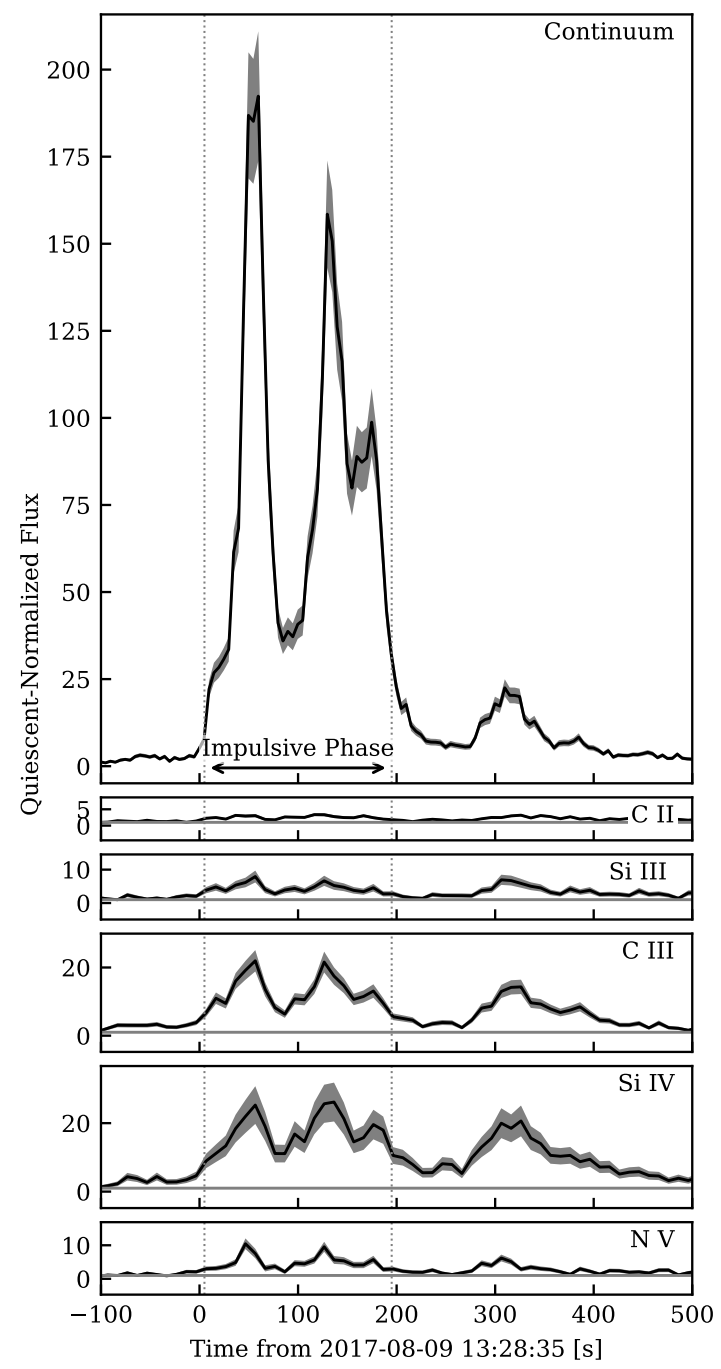

Figure 3. Lightcurves of major emission lines and continuum regions for the Hazflare normalized by their quiescent flux. Dotted lines bracket the impulsive phase that was integrated to produce the spectrum in Figure 5. Sizes of the axes have been adjusted such that the scales are closely similar. All lines are the same as those identified in Figure 5 with multiple components coadded, except that $\mathrm{C}$ III refers only to the multiplet at $1175 \AA$, not the line at $1247 \AA$. Horizontal lines at unity are shown to guide the eye.

The most energetic flare observed on the Tuc-Hor objects was a superflare (Schaefer et al. 2000), meaning its bolometric energy exceeded $10^{33} \mathrm{erg}$, more than any recorded solar flare. We have dubbed this event the Hazflare. The Hazflare occurred on the M2.0 object GSC 8056-0482 (J02365 in this work), peaking at 2017 August 09 13:29 UT and is, to our knowledge, the most energetic stellar flare yet observed in the FUV by HST. Lightcurves and spectra for this flare are plotted in Figures 3, 4, and 5. Confident classification of this event as a superflare is possible due to a well-resolved black-

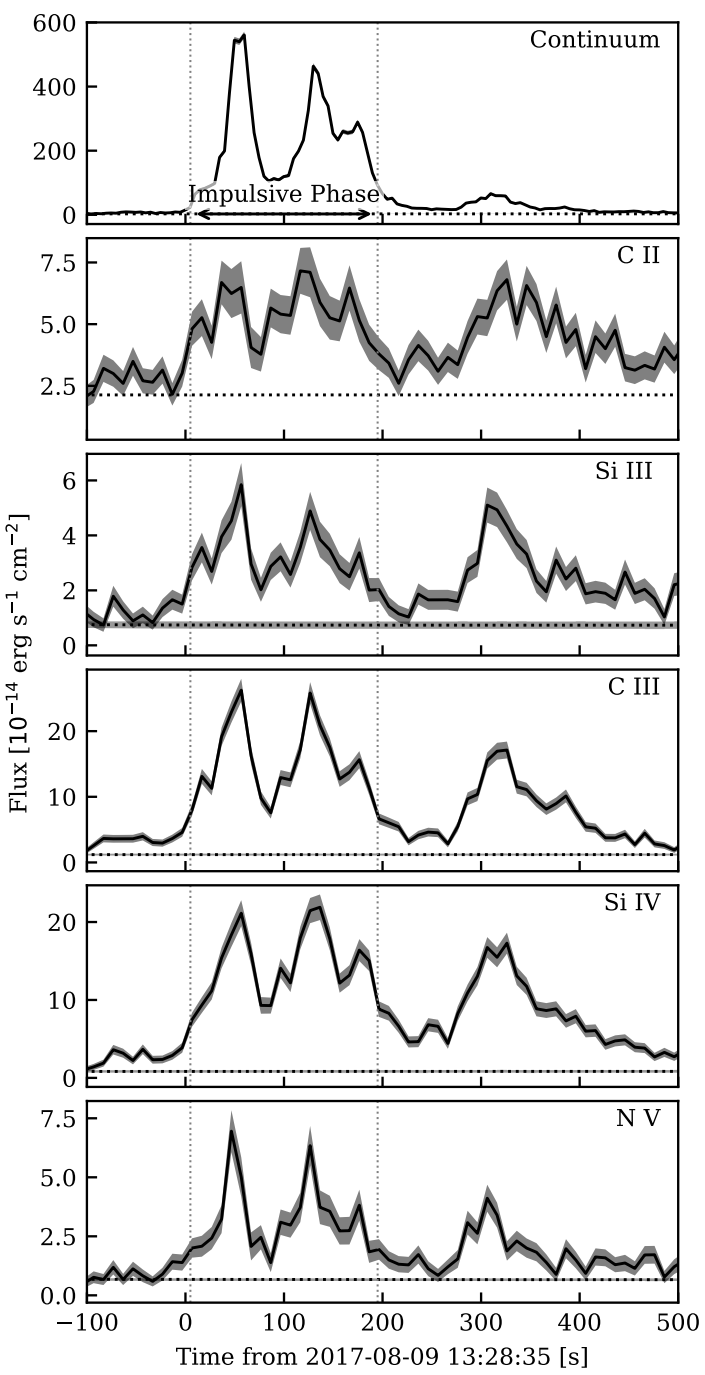

Figure 4. Lightcurves of major emission lines and continuum regions for the Hazflare in absolute flux. The best-fit continuum and uncertainty is shown as the dotted horizontal line. Dotted vertical lines bracket the impulsive phase that was integrated to produce the spectrum in Figure 5 .

body continuum in the FUV that can be extrapolated across all wavelengths as a lower limit on the bolometric flare energy. A fit to this continuum, shown in Figure 6 , implies the blackbody had a characteristic temperature of $15,500 \pm 400 \mathrm{~K}$ across the impulsive phase of the flare and alone emitted a wavelength-integrated energy of $10^{33.44 \pm 0.04} \mathrm{erg}$ over the entire flare. In the $U$ band, commonly used for ground-based flare observations, the blackbody emitted $10^{32.47 \pm 0.05} \mathrm{erg}$. Including an appropriately scaled version of the fiducial flare energy budget in L18, which includes estimates of nonthermal emission in the unobserved FUV and inobservable EUV, increases the bolometric energy estimate to $10^{33.6_{-0.2}^{+0.1}} \mathrm{erg}$ (where 
we have assumed an order-of-magnitude uncertainty in the EUV scalings).

This observation is of particular value because superflares are common on stars (e.g., Davenport 2016), yet spectrophotometry of such flares in the UV, the band most relevant to planetary atmospheric photochemistry, is rare. Superflares are estimated from Kepler data to occur on M0-M4 dwarfs at a frequency of a few per day (Yang et al. 2017). Photochemical models exploring the effects of flares on planetary atmospheres have thus far relied primarily on observations of the 1985 Great Flare on AD Leo (Hawley \& Pettersen 1991; Segura et al. 2010; Tilley et al. 2017), a flare estimated to emit a bolometric energy of $10^{34} \mathrm{erg}$. The Great Flare also showed a clear continuum in FUV emission, and overall the continuum was responsible for at least an order of magnitude more overall energy emitted by the flare than lines, consistent with the Hazflare. However, the Great Flare observations, made with the International Ultraviolet Explorer, saturated in the strongest emission lines, degrading their accuracy. The present observation clearly resolved the temporal evolution in all major emission lines over the course of the flare, except for Ly $\alpha$ and O I since these are contaminated by Earth's geocoronal emission.

Major emission lines during the Hazflare are shown in Figure 7. The lines show redshifts from $50-80 \mathrm{~km} \mathrm{~s}^{-1}$ similar to previous observations of FUV lines during flares (e.g., Hawley et al. 2003, L18), that signify downward flows of material during the flare. During the flare, a strong line appears at $1247 \AA$ of which there is no hint in the star's quiescent spectrum. Based on the CHIANTI solar atmosphere model (Dere et al. 2009), we identify this line to be a transition of C III.

The C III $1247 \AA$ line is dipole allowed, with a similar transition probability to the components of the $\mathrm{C}$ III complex at $1175 \AA$ (not shown in Figure 7 because of blending). However, the upper level of the $1247 \AA$ transition is $5.6 \mathrm{eV}$ more energetic, hence it is more difficult to populate. The distinct on/off nature of this line between the flaring and quiescent state could be an important constraint on the physical conditions elicited by the flare. A possible explanation is that the upper level of the $1247 \AA$ transition is populated by collisional excitation from the upper level of the $1175 \AA$ transitions. Because radiative de-excitation to produce the $1175 \AA$ lines is fast, a threshold rate of collisions would need to be reached to excite to the upper level of the $1247 \AA$ transition. However, such excitation might then quench the $1175 \AA$ line, and this is not observed. We encourage further exploration of this topic in future modeling work.
The spectral energy budget of the Hazflare is within the scatter in energy budgets of $\mathrm{M}$ dwarf flares of lower energy as analyzed by L18. The most important feature, energetically, of the flare spectrum is the blackbody. Multiwavelength observations of AD Leo flares by Hawley et al. (2003) that all exhibited roughly $9000 \mathrm{~K}$ emission yielded ratios of blackbody emission to Si IV emission of 100-200. In comparison, this ratio for the $15,500 \mathrm{~K}$ Hazflare is 240. This could be an important constraint for simulations of flares, as it suggests the partitioning of energy between FUV and blackbody emission remains relatively constant even though one might reasonably expect the relative contribution of the blackbody to be a factor of $(15,000 / 9,000)^{4} \approx 8$ higher in a $15,000 \mathrm{~K}$ versus a $9,000 \mathrm{~K}$ flare.

The Hazflare is not of exceptional FUV energy when normalized by the quiescent luminosity of the star, i.e., when energy is quantified as equivalent duration. Several HST observations of other $\mathrm{M}$ dwarfs yielded flares of greater FUV equivalent duration, specifically those of $15 \mathrm{ks}$ and $12 \mathrm{ks}$ on Prox Cen and 6.8 ks on GJ 876 (L18). The temporal evolution of the Hazflare is similar to these events, exhibiting multiple rapid increases and drops in emission, the "impulsive phase" of the flare. (Note some other authors reserve the use of the term "impulsive" for the initial flare rise, e.g., Bookbinder et al. 1992.)

This structure is also qualitatively mimicked in the integrated $U$-band flux of the Great AD Leo Flare. However, the impulsive phase of the Great AD Leo Flare lasts about $4 \times$ longer than the Hazflare. If the two flares have similar continuum emission, this alone could potentially explain the greater energy of the Great Flare. Hawley \& Pettersen (1991) took the continuum radiation of the Great AD Leo Flare to be that of recombination continua and unresolved lines and did not estimate blackbody temperatures or filling factors.

In determining the temperature of the Hazflare blackbody, we addressed the effect of extinction by the ISM. Extinction is much stronger at FUV wavelengths than the $U$ band wavelengths where blackbody emission is usually resolved, and the $41.7 \mathrm{pc}$ distance to this star could potentially contain sufficient dust for significant absorption. Using the 3D local ISM dust model, we estimated a worst-case $E(B-V)$ extinction of 0.015 mag. As a worst-case $R_{V}$ (a parameter that sets the relationship between extinction and wavelength), we take a value of 2 given at least one known sight line has an $R_{V}$ near this extreme: 2.1 for HD 210121 (Welty \& Fowler 1992). This yielded a predicted absorption of $10 \%-7 \%$ across G130M bandpass and increased the temperature of the best-fit blackbody by $300 \mathrm{~K}$. We considered this 


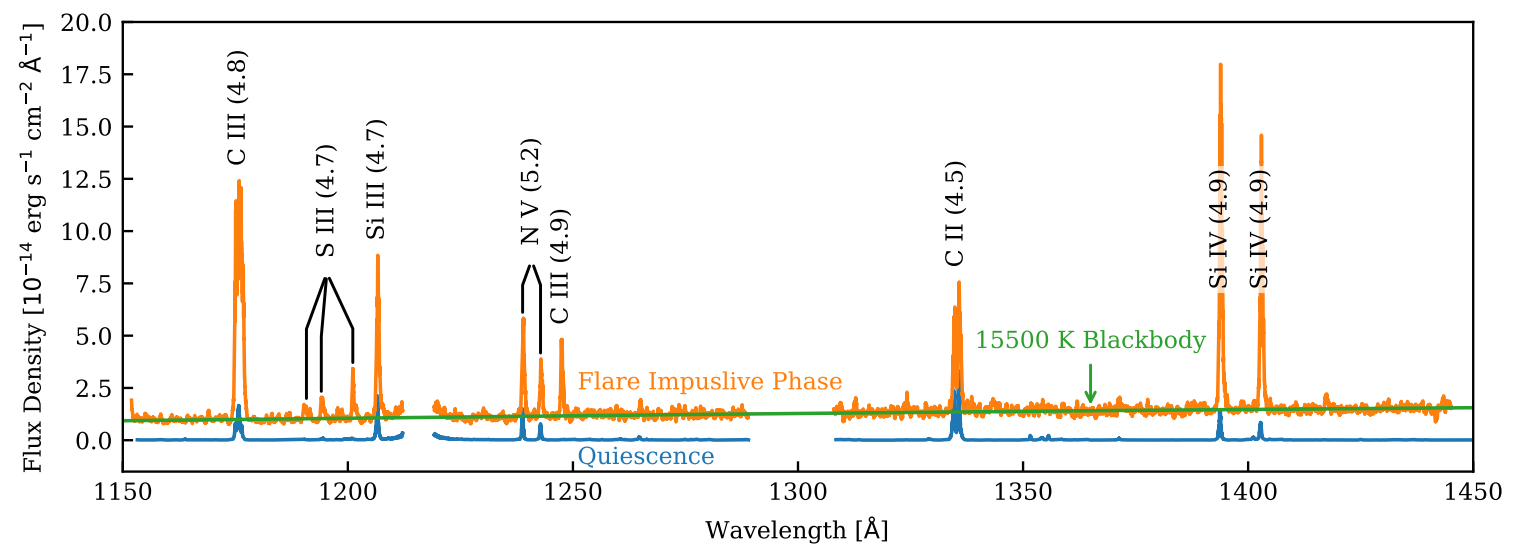

Figure 5. The spectrum of the Hazflare averaged over its impulsive phase (orange; Figure 3 shows integration limits) with the best-fit blackbody overplotted (green) and compared to the quiescent spectrum of the star (blue). Major lines are identified with the log formation temperature (in Kelvin) from a model solar atmosphere (Dere et al. 2009) shown in parenthesis. Regions within the COS detector gap and regions contaminated by geocoronal emission have been removed.

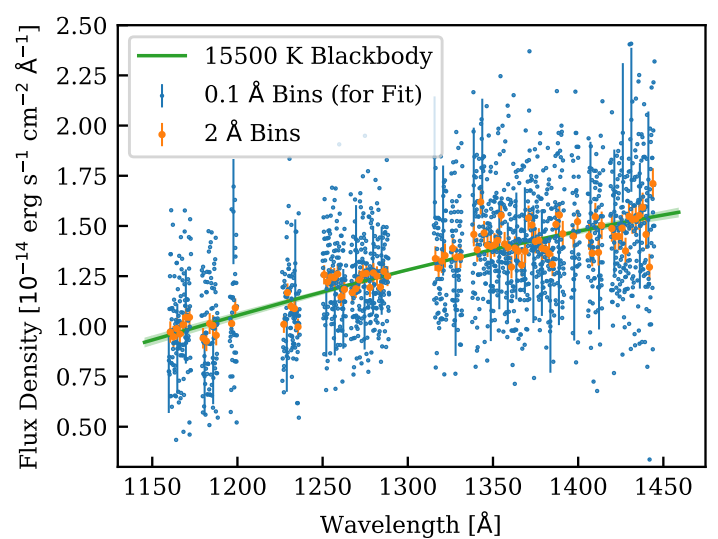

Figure 6. Blackbody fit to the continuum regions of the Hazflare spectrum during its impulsive phase.

effect minimal and this scenario unlikely, so we neglected reddening in the remainder of our analysis.

The $15,500 \mathrm{~K}$ temperature of the Hazflare blackbody is somewhat uncommon, above the 9,000-14,000 K range observed by Kowalski et al. (2013) (though they estimate up to $\sim 1000 \mathrm{~K}$ uncertainties). However, GALEX observations of a flare on GJ 3685A (M4) suggest a blackbody temperature of 50,000 K based on the ratio of broadband FUV to NUV flux (Robinson et al. 2005) and blue-optical observations of continuum emission from flares on YZ CMi (also M4) could be consistent with plasma temperatures as high as $170,000 \mathrm{~K}$ (Kowalski et al. 2013; ?). Flare modeling assuming heating by a beam of nonthermal electrons directed at the stellar surface by Kowalski et al. (2015) has successfully reproduced blackbody-like emission $\approx 10,000 \mathrm{~K}$ in the blue optical. Earlier models using lower electron beam energy fluxes could not reproduce this emission, suggesting that further increasing the energy flux in such models might reproduce the Hazflare continuum emission. It is noteworthy that the the continuum shape in these models, though it is blackbody-like, is more attributable to changing optical depth with wavelength than the temperature of an optically thick source.

To the knowledge of the authors, the Hazflare is the first event in which a blackbody emission temperature could be constrained using FUV spectra. It is possible even higher temperature emission is present in some flares, but is not well-constrained by $U$ band spectra since they are further within the Rayleigh-Jeans tail of the Planck curve. Future flare observations in the FUV and NUV could reveal more events like the Hazflare or hotter.

High blackbody temperatures were sustained throughout the Hazflare. In Figure 8, we show the temperature of the blackbody in three separate time intervals (limited signal precludes further divisions in time). The flare peaks initially nearer to $16,000 \mathrm{~K}$ and decays to $14,000 \mathrm{~K}$. We plot the filling factor of the flare in Figure 8 as well, a quantity specifying the area of blackbody emission required to yield the flare continuum flux as a fraction of the star's visible hemisphere. In comparison to the AD Leo observations of Hawley et al. (2003); Kowalski et al. (2013), the high temperature of the Hazflare yields a comparatively small filling factor despite a larger flare energy. Along with the hotter flares characterized in Kowalski et al. (2013), the Hazflare lends support to the idea that heating area, duration, and intensity are all important in determining the total energy emitted by a flare, rather than only area and duration.

During the Hazflare, the covering fraction increases with each successive peak in the triple-peaked flare, yet 


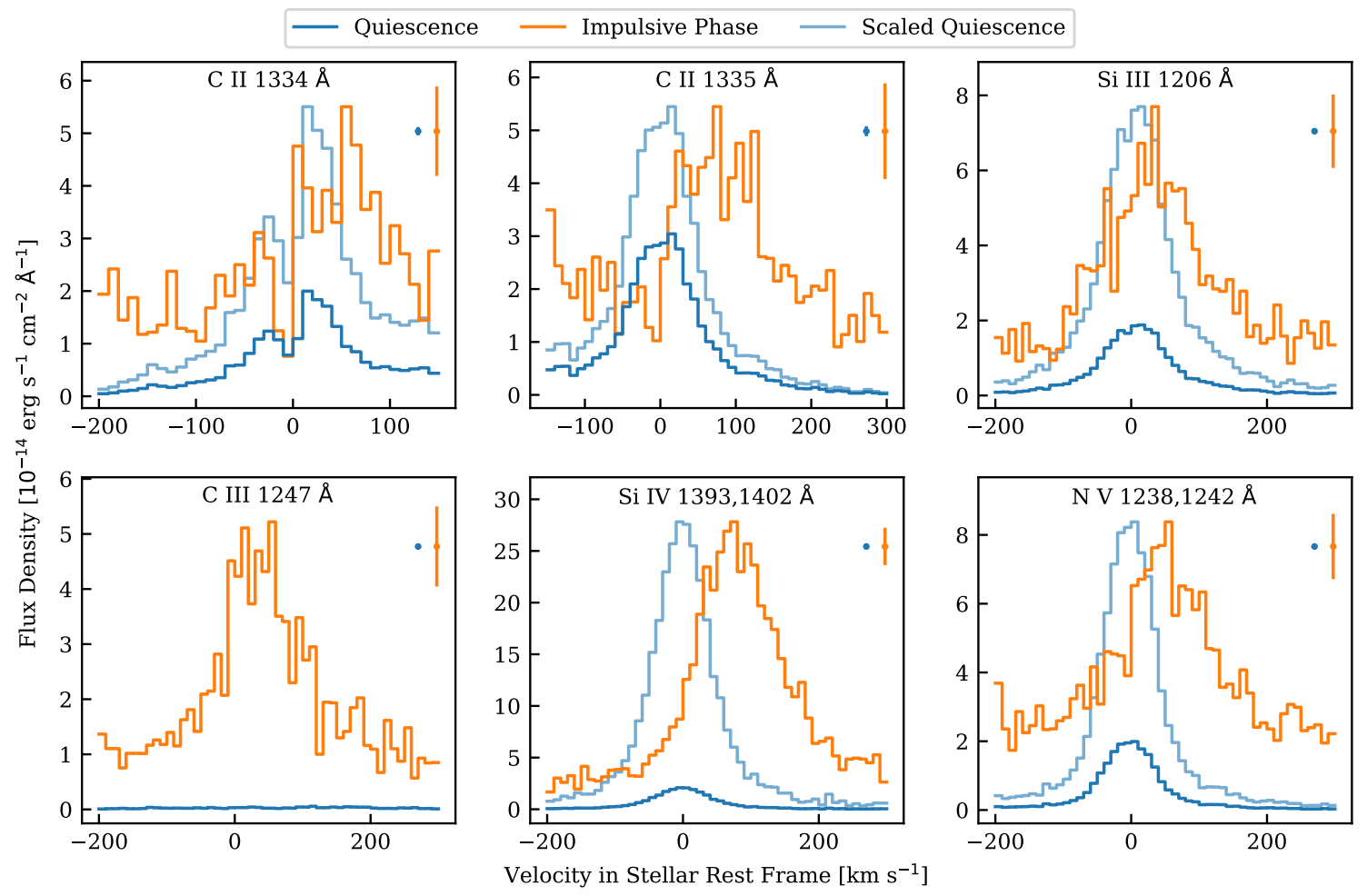

Figure 7. Major emission lines during the Hazflare versus quiescence. Dark blue shows the quiescent line profile, orange the profile during the impulsive phase of the Hazflare, and light blue is a scaled quiescent profile to facilitate the comparison of line shapes (not shown for C III since no line is resolved in quiescence). The N V and Si IV doublets are coadded, but this is not done for C II due to ISM absorption affecting one component. The spectra are binned to $10 \mathrm{~km} \mathrm{~s}^{-1}$ resolution, roughly the absolute wavelength accuracy of COS. Bars representative of the error at the line peaks are shown on the top right of each panel.

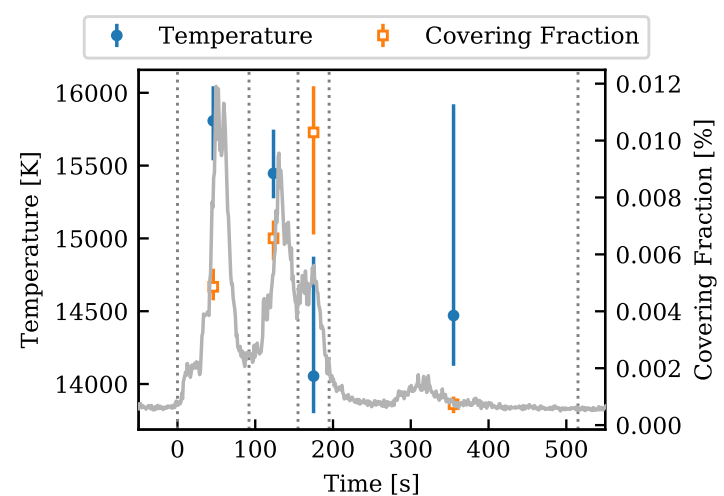

Figure 8. Evolution of blackbody emission over the course of the Hazflare. Dashed vertical lines delineate the integration ranges used to produce spectra for Planck-function fits. The lightcurve of emission in the continuum bands is plotted as well for reference.

the successive drops in blackbody temperature result in a lower energy emitted by each peak. Similar behavior was present in a flare on the M dwarf YZ CMi observed by Mochnacki \& Zirin (1980). However, evolution of the blackbody during flares does not seem to follow a predictable pattern. Temperature and covering fraction evolved in lock-step during a YZ CMi flare observed by Kowalski et al. (2013). In several flares on the M dwarf AD Leo observed by Hawley et al. (2003), temperature and covering fraction sometimes track well, and sometimes do not. When they do not, emission closely tracks covering fraction rather than temperature. For the Hazflare temperature and covering fraction do not track, and temperature appears to better account for changes in emission.

\subsection{Planetary Implications of the Hazflare}

Flares like the Hazflare will bombard orbiting planets, influencing the immediate state as well as long-term evolution of their atmospheres. For the young M2.0 star that produced the Hazflare, the eventual main-sequence HZ lies 0.1-0.2 AU from the star (adopting a stellar mass of $0.4 M_{\odot}$; Kopparapu et al. 2013). Note that a planet orbiting in this range will be too hot to support liquid water currently because the star is about twice as luminous at its present $40 \mathrm{Myr}$ age as it will be several Gyr in the future, i.e. the $\mathrm{HZ}$ will move inward by about $40 \%$ as the star evolves onto the main sequence, according to the evolutionary tracks of Baraffe et al. (2015). The decline in luminosity that drives the HZ evolution 
is predicted to last until $250 \mathrm{Myr}$, then over the next 10 Gyr the star's luminosity is predicted to increase by $9 \%$.

Because the 9,000-14,000 K range of flare blackbodies thus far observed peak in the NUV, they are wellsuited to photolyzing ozone. Ozone has a peak in its photolysis cross section at $2550 \AA$, corresponding to an $11,400 \mathrm{~K}$ blackbody. Varying the blackbody temperature of equal-energy flares from 5,000-15,000 K can change the photolysis rate $\left(J\right.$-value) of unshielded $\mathrm{O}_{3}$ molecules, by a factor of a few (L18). During the impulsive phase of the Hazflare, the blackbody emission could drive $J_{\mathrm{O}_{3}}=0.04 \mathrm{~s}^{-1}$ for a planet at mid-HZ, about $5 \times$ that of the Sun at Earth (Loyd et al. 2016).

The recombination of $\mathrm{O}$ and $\mathrm{O}_{2}$ is very fast, as is the thermal dissociation of $\mathrm{O}_{3}$. In consequence, $\mathrm{O}$ and $\mathrm{O}_{3}$ are rapidly exchanged, quickly reaching an equilibrium ratio under a given set of conditions. This ratio is driven by the NUV radiation field, so as the NUV flux of a flare evolves, the $\mathrm{O}_{3}$ column evolves almost in lock-step. Ultimately, the dissociation of $\mathrm{O}_{2}$ by the flare's FUV radiation will provide additional atomic oxygen reservoir that, once the elevated NUV flux abates, yields an $\mathrm{O}_{3}$ column that is greater than at the flare onset. This phenomenon is clear in the simulations of Segura et al. (2010) and Tilley et al. (2017) when only EM radiation from flares is considered. If energetic particles are assumed to accompany a flare, they produce $\mathrm{O}_{3}$-destroying catalysts that then drive a dramatic depletion of $\mathrm{O}_{3}$ over timescales well beyond the duration of any single flare. For the Hazflare, applying the solar scaling of Youngblood et al. (2017) yields an estimate for the fluence of $>10 \mathrm{MeV}$ protons in the main-sequence $\mathrm{HZ}$ of $10^{5.6 \pm 0.8}$ proton $\mathrm{cm}^{-2} \mathrm{~s}^{-1} \mathrm{sr}^{-1}$, two orders of magnitude above the largest solar observation given in Youngblood et al. (2017).

Of course, an atmosphere need not be Earth-like, and flares could be an important photolyzer of other molecules. Noteworthy is the methane prevalent in Titan's atmosphere whose photolysis products go on to assemble long hydrocarbon chains that contribute to an atmospheric haze (see Hörst 2017 for a recent review). As with ozone, secondary catalytic reactions are important here, as $\mathrm{C}_{2} \mathrm{H}_{2}$ and $\mathrm{C}_{4} \mathrm{H}_{2}$ further destroy $\mathrm{CH}_{4}$ once they are produced from its photolysis products. Similarly, work by $\mathrm{Hu}$ et al. (2013) has shown that in reducing atmospheres, photolysis of surface-outgassed $\mathrm{H}_{2} \mathrm{~S}$ and $\mathrm{SO}_{2}$ species can yield $\mathrm{S}$ and $\mathrm{S}_{2}$ that then polymerize into hazes. In these instances, flares like the Hazflare could be an important additional source term for producing haze-forming monomers. Hazes are important to exoplanet observations because they could obscure absorption features in transmission spectroscopy (e.g., Kreidberg et al. 2014; Kawashima \& Ikoma 2018). They are also important to life, as they could shield the surface from UV radiation and might also act as a source of biological precursor molecules (Hu et al. 2013).

Similar to ozone, the photolysis cross section of $\mathrm{H}_{2} \mathrm{~S}$ has a peak in the NUV. However, photolysis cross sections for $\mathrm{CH}_{4}$ are skewed more toward the FUV. Thus differences in the blackbody temperature of a flare can have a more dramatic effect on this molecule (L18). During the impulsive phase of the flare, the Hazflare blackbody alone could drive $J_{\mathrm{CH}_{4}}=0.01 \mathrm{~s}^{-1}$ at mid-HZ, $1600 \times$ that of the Sun at $1 \mathrm{AU}$.

Another relevant feature of the Hazflare's 15,500 K blackbody is emission in the EUV at wavelengths where photons can ionize H. Such emission would heat the upper atmospheres of orbiting planets, potentially powering additional thermal atmospheric escape from the planet. The blackbody emits an energy in the 100-912 $\AA$ EUV range equivalent to $2 \%$ of that given by the fiducial flare of L18 scaled to match the Hazflare's Si IV emission. The contribution of EUV energy to the fiducial flare is based on scaling from solar data (see L18 for details).

Energy conservation requires that the EUV (blackbody and otherwise) irradiation of the Hazflare could not have removed more than $\sim 10^{12} \mathrm{~g}$ of atmospheric mass from an Earth-gravity planet at mid-HZ, $\sim 10^{-9}$ the mass of Earth's atmosphere. The fact that the relatively brief ( $35 \mathrm{ks}$ ) cumulative exposure of the HAZMAT campaign captured such a flare suggests that they are common. These observations constrain the rate of flares emitting $>10^{33} \mathrm{erg}$ in the FUV (i.e., FUV superflares) to $0.1-1 \mathrm{~d}^{-1}$ (Table 5). If the mass-loss efficiency for flares is similar to the canonical value of 0.1 for steadystate flux (e.g., Murray-Clay et al. 2009), then the accumulated atmospheric "erosion" by such flares could be significant over timescales of hundreds of Myr. Chadney et al. (2017) modeled the effects of a flare on mass loss from hot Jupiters and found that it could not explain variations seen in the Ly $\alpha$ transit of HD 189733b. More modeling is needed to determine the efficiency of flare EUV emission in removing atmospheric mass over a range of planetary parameters (e.g. mass), physical regimes of mass loss, and flare energies. In addition, particle events associated with $\mathrm{M}$ star flares are a persistent unknown. If they accompany highly energetic events as on the Sun such as predicted by the scaling relations of Youngblood et al. (2017), then flare erosion of planetary atmospheres would be more severe.

\section{SUMMARY AND FUTURE WORK}



of young M dwarfs, we identified and analyzed FUV flares that occurred on a sample of $12 \mathrm{M} 0-\mathrm{M} 2.3$ stars in the Tuc-Hor association, age $40 \mathrm{Myr}$, during $35.5 \mathrm{ks}$ of HST COS-G130M observations. We identified 18 flares in total and fit a power law to the distribution of these flares.

These young $\mathrm{M}$ stars are indeed more active in terms of flares than a comparison sample of older, inactive field M1.5-M3.5 dwarfs for which an identical flare analysis was carried out by L18. Specifically, on the $40 \mathrm{Myr}$ M stars flares with energy $>10^{30}$ erg occur $20-100 \times$ more frequently than on the field-age M stars. Alternatively, the limiting energy for flares occurring at a rate of $<5 \mathrm{~d}^{-1}$ is $100-1000 \times$ greater. This elevation in flare activity at young ages mimics that of the average emission in the GALEX FUV and NUV bands (Shkolnik \& Barman 2014; Schneider \& Shkolnik 2018).

When the flare distributions are specified in equivalent duration rather than absolute energy, a metric that normalizes the flare energy by the stars' quiescent luminosities in the same band, we find the distributions of the $40 \mathrm{Myr}$ and field-age M stars closely overlap. This complements the finding of L18 that active versus inactive $\mathrm{M}$ stars show no significant difference in FUV flare activity when equivalent duration is used to characterize their flares, extending it to old versus young samples as well. In addition, the power-law fit to the equivalent duration distribution implies that more overall energy will be emitted in the FUV from flares versus quiescence if that power-law extends another $1-5$ orders of magnitude beyond the most energetic flare in the sample.

The most energetic flare that occurred during the observations was a superflare (bolometric energy $>10^{33}$ erg). A strong lower limit can be placed on the bolometric flux of this flare because of the greatly elevated continuum emission manifested by the flare, nearly $200 \times$ quiescent levels. This made the continuum bright enough for its slope and curvature to be clearly resolved, permitting a blackbody fit that implies $15,500 \pm 400 \mathrm{~K}$ emission was responsible for this continuum emission over the flare. Stitching together the measured emission, blackbody fit, and the fiducial flare template of L18, we estimate a bolometric energy of this flare of
With the aim of constraining the FUV flare activity

$10^{33.6_{-0.2}^{+0.1}} \mathrm{erg}$, approaching the estimated $10^{34} \mathrm{erg}$ of the 1985 Great Flare on AD Leo. The hot blackbody emission of this flare would be a powerful photolyzer of most molecules in planetary atmospheres due to the high broadband FUV flux.

This and previous UV flare analyses beg further study and observations in several key areas. Whether flares actually dominate quiescent emission is a question that cannot be confidently resolved until there are observations in the UV of sufficient cumulative time to constrain the high-energy tail of the FFD. If the FFD for FUV flares on $40 \mathrm{My} \mathrm{M}$ stars obeys Eqn. 3 out to $10^{36} \mathrm{erg}$ flares (close to the maximum observed by Kepler; Yang et al. 2017), these stars would need to be observed for several months to obtain meaningful constraints on the rate of such flares. Additional observations are needed to fully characterize the diversity of continuum flux among flares, as the blackbody temperature of this emission is critical to photochemical models. Further modeling work is needed in the area of flare erosion of planetary atmospheres to asses the (in)significance of this erosion in driving the lifelong evolution of planetary atmospheres. Finally, diagnostics of particle events associated with stellar flares are imperative, as particles likely have much more severe implications for planetary atmospheres than flare EM radiation, yet their severity and even existence in relation to M-star flares has yet to be strongly observationally constrained.

R.O.P.L. and E.S. gratefully acknowledge support from NASA HST Grant HST-GO-14784.001-A for this work. R.O.P.L. thanks Brittany Miles, Adam Kowalski, Wilson Cauley, Melodie Kao, Tyler Richey-Yowell, Ella Osby, and Allison Youngblood for helpful discussions relating to this work.

\section{Facilities: Hubble Space Telescope}

Software: extinction (v0.3.0, Barbary et al. 2016, http://extinction.readthedocs.io/) emcee (v2.2.0, Foreman-Mackey et al. 2013, http: //dfm.io/emcee/current/) celerite (v0.3.0, Foreman-Mackey et al. 2017, http://celerite. readthedocs.io) FLAIIL (Loyd et al. 2018, https: //github.com/parkus/flaiil), FFD (Loyd et al. 2018, https://github.com/parkus/ffd)

\section{REFERENCES}

Anglada-Escudé, G., Tuomi, M., Gerlach, E., et al. 2013, A\&A, 556, A126

Baraffe, I., Homeier, D., Allard, F., \& Chabrier, G. 2015, A\&A, 577, A42
Barbary, K., Barclay, T., Biswas, R., et al. 2016, SNCosmo:

Python library for supernova cosmology, Astrophysics

Source Code Library, , , ascl:1611.017 
Bell, C. P. M., Mamajek, E. E., \& Naylor, T. 2015, MNRAS, 454, 593

Berta, Z. K., Charbonneau, D., Bean, J., et al. 2011, ApJ, 736,12

Bookbinder, J. A., Walter, F. M., \& Brown, A. 1992, in Cool Stars, Stellar Systems, and the Sun, ed. M. S. Giampapa \& J. A. Bookbinder, Vol. 26, 27

Chadney, J. M., Koskinen, T. T., Galand, M., Unruh, Y. C., \& Sanz-Forcada, J. 2017, A\&A, 608, A75

Davenport, J. R. A. 2016, ApJ, 829, 23

Davenport, J. R. A., Kipping, D. M., Sasselov, D., Matthews, J. M., \& Cameron, C. 2016, ApJ, 829, L31

Dere, K. P., Landi, E., Young, P. R., et al. 2009, A\&A, 498, 915

Fleming, B. T., France, K., Nell, N., et al. 2018, Journal of Astronomical Telescopes, Instruments, and Systems, 4, 014004

Foreman-Mackey, D., Agol, E., Ambikasaran, S., \& Angus, R. 2017, AJ, 154, 220

Foreman-Mackey, D., Hogg, D. W., Lang, D., \& Goodman, J. 2013, Publications of the Astronomical Society of the Pacific, 125, 306

France, K., Loyd, R. O. P., Youngblood, A., et al. 2016a, ApJ, 820, 89

-. 2016b, ApJ, 820, 89

Hall, J. C. 2008, Living Reviews in Solar Physics, 5, 2

Hawley, S. L., Davenport, J. R. A., Kowalski, A. F., et al. 2014, ApJ, 797, 121

Hawley, S. L., \& Pettersen, B. R. 1991, ApJ, 378, 725

Hawley, S. L., Allred, J. C., Johns-Krull, C. M., et al. 2003, ApJ, 597, 535

Hilton, E. J. 2011, PhD thesis, University of Washington

Hörst, S. M. 2017, Journal of Geophysical Research (Planets), 122, 432

Howard, W. S., Tilley, M. A., Corbett, H., et al. 2018, ApJ, 860, L30

Hu, R., Seager, S., \& Bains, W. 2012, ApJ, 761, 166

-. 2013, ApJ, 769, 6

Kawashima, Y., \& Ikoma, M. 2018, ApJ, 853, 7

Kjeldsen, H., \& Bedding, T. R. 1995, A\&A, 293, 87

Kopparapu, R. K., Ramirez, R., Kasting, J. F., et al. 2013, ApJ, 765, 131

Kowalski, A. F., Hawley, S. L., Carlsson, M., et al. 2015, SoPh, 290, 3487

Kowalski, A. F., Hawley, S. L., Wisniewski, J. P., et al. 2013, The Astrophysical Journal Supplement Series, 207, 15

Kraus, A. L., Shkolnik, E. L., Allers, K. N., \& Liu, M. C. 2014, AJ, 147, 146
Kreidberg, L., Bean, J. L., Désert, J.-M., et al. 2014, Nature, 505, 69

Lammer, H., Selsis, F., Ribas, I., et al. 2003, ApJ, 598, L121

Loyd, R. O. P., \& France, K. 2014, The Astrophysical Journal Supplement Series, 211, 9

Loyd, R. O. P., France, K., Youngblood, A., et al. 2016, ApJ, 824, 102

—. 2018, ArXiv e-prints, arXiv:1809.07322

Maeder, A., \& Mermilliod, J. C. 1981, A\&A, 93, 136

Martín, E. L., Lodieu, N., Pavlenko, Y., \& Béjar, V. J. S. 2018, ApJ, 856, 40

Maschberger, T., \& Kroupa, P. 2009, MNRAS, 395, 931

Miguel, Y., \& Kaltenegger, L. 2014, ApJ, 780, 166

Miles, B. E., \& Shkolnik, E. L. 2017, AJ, 154, 67

Mochnacki, S. W., \& Zirin, H. 1980, ApJ, 239, L27

Morrissey, P., Conrow, T., Barlow, T. A., et al. 2007, The Astrophysical Journal Supplement Series, 173, 682

Murray-Clay, R. A., Chiang, E. I., \& Murray, N. 2009, ApJ, 693, 23

Perryman, M. A. C., Brown, A. G. A., Lebreton, Y., et al. 1998, A\&A, 331, 81

Rivera, E. J., Laughlin, G., Butler, R. P., et al. 2010, ApJ, 719,890

Robinson, R. D., Wheatley, J. M., Welsh, B. Y., et al. 2005, ApJ, 633, 447

Sanz-Forcada, J., Ribas, I., Micela, G., et al. 2010, A\&A, 511, L8

Schaefer, B. E., King, J. R., \& Deliyannis, C. P. 2000, ApJ, 529, 1026

Schneider, A. C., \& Shkolnik, E. L. 2018, AJ, 155, 122

Segura, A., Walkowicz, L. M., Meadows, V., Kasting, J., \& Hawley, S. 2010, Astrobiology, 10, 751

Selsis, F., Kasting, J. F., Levrard, B., et al. 2007, A\&A, 476, 1373

Shields, A. L., Ballard, S., \& Johnson, J. A. 2016, PhR, 663,1

Shkolnik, E. 2016, Monitoring the High-Energy Radiation Environment of Exoplanets around Lowmass Stars with SPARCS (Star-Planet Activity Research CubeSat), NASA APRA Proposal, ,

Shkolnik, E. L., Anglada-Escudé, G., Liu, M. C., et al. 2012, ApJ, 758, 56

Shkolnik, E. L., \& Barman, T. S. 2014, AJ, 148, 64

Shkolnik, E. L., Liu, M. C., Reid, I. N., Dupuy, T., \& Weinberger, A. J. 2011, ApJ, 727, 6

Tilley, M. A., Segura, A., Meadows, V. S., Hawley, S., \& Davenport, J. 2017, ArXiv e-prints, arXiv:1711.08484

Torres, C. A. O., Quast, G. R., da Silva, L., et al. 2006, A\&A, 460, 695 
Torres, G., Winn, J. N., \& Holman, M. J. 2008, ApJ, 677, 1324

Welsh, B. Y., Wheatley, J. M., Seibert, M., et al. 2007, The Astrophysical Journal Supplement Series, 173, 673

Welty, D. E., \& Fowler, J. R. 1992, ApJ, 393, 193
Yang, H., Liu, J., Gao, Q., et al. 2017, ApJ, 849, 36

Youngblood, A., France, K., Loyd, R. O. P., et al. 2017, ApJ, 843, 31 